\title{
Permittivity measurement without calibration in the waveguide-penetration method
}

\author{
Alfred Kik ${ }^{1}$ and Atsuhiro Nishikata ${ }^{2}$ \\ ${ }^{1}$ Department of Electrical and Electronic Engineering, Tokyo Metropolitan \\ University, 1-1 Minamiosawa, Hachioji-shi, Tokyo 192-0397, Japan \\ 2 Department of Human System Science, Tokyo Institute of Technology, \\ 2-12-1 Ookayama, Meguro-ku, Tokyo 152-8552, Japan
}

\begin{abstract}
We present a new approach for the broadband characterization of dielectric materials in the waveguide-penetration method. The new approach uses only two sets of uncalibrated S-parameter measurements: when the dielectric material is loaded into the waveguide, and when it is unloaded. The theory behind the new approach is based on the properties of similar matrices arising during the measurement. Compared to the previous methods, a network analyzer calibration is not required anymore, resulting in a great measurement simplification. We perform measurements in the X-band to validate the new approach.
\end{abstract}

Keywords: complex permittivity measurement, rectangular waveguide, cylinder, S-parameter, calibration

Classification: Electromagnetic Compatibility (EMC)

\section{References}

[1] R. Clarke and A. Gregory, "A guide to the characterization of dielectric materials at RF and microwave frequencies," NPL Report, 2003.

[2] J. Baker-Jarvis, "Transmission/Reflection and short-circuit line permittivity measurements," NIST Tech. Note, 1990.

[3] A. Nishikata, "Scattering analysis for layered cylindrical object perpendicularly piercing the wider walls of a rectangular waveguide and its application to $\varepsilon_{r}$ and $\mu_{r}$ measurement," IEEE Trans. Microw. Theory Tech., vol. 57, no. 6, pp. 1602-1611, Jun. 2009. DOI:10.1109/TMTT.2009.2020838

[4] G. Engen and C. Hoer, "Thru-Reflect-Line: an improved technique for calibrating the dual six-port automatic network analyzer," IEEE Trans. Microw. Theory Tech., vol. 27, no. 12, pp. 987-993, Dec. 1979. DOI:10.1109/TMTT.1979.1129778

[5] A. Kik and A. Nishikata, "A new calibration algorithm using reference materials for the waveguide-penetration method," IEICE Trans. Commun., vol. E94-B, no. 9, pp. 2549-2557, Sep. 2011. DOI:10.1587/transcom.E94.B.2549

[6] R. B. Marks, "Formulations of the basic vector network analyzer error model including switch-terms," ARFTG Conf. Dig. Fall, 50th, vol. 32, pp. 115-126, Dec. 1997. DOI:10.1109/ARFTG.1997.327265

[7] R. A. Horn and C. R. Johnson, "Similarity," in Matrix Analysis, pp. 44-57, Cambridge University Press, 1990. 


\section{Introduction}

In EMC and microwave engineering, it is important to know the electromagnetic properties of materials such as their complex permittivity. For this purpose, several characterization methods have been developed over the years [1]. The conventional swept-frequency transmission/reflection (T/R) method [2] is shown in Fig. 1(a). In this method, the material under test (MUT) is rectangular, and it fills completely the cross section of a rectangular waveguide. The complex permittivity of the material is extracted from the S-parameters that are measured by a calibrated vector network analyzer (VNA). The week points of this method are the difficulty of fabricating the MUT in a rectangular shape, the difficulty of inserting the MUT into the waveguide, and the need of VNA calibration prior to measurement.

With the simplification of the measurement process in mind, Nishikata proposed in [3] a new T/R method: the waveguide-penetration method. As shown in Fig. 1(b), this method uses a cylindrical MUT which can be easily fabricated. In addition, the MUT can be easily inserted into the waveguide through a pair of holes in the waveguide walls. In the same manner as the conventional $T / R$ methods, the waveguide-penetration method still measures calibrated S-parameters to extract the complex permittivity of the material. The system is usually calibrated by means of the conventional TRL method [4] or by means of two or more reference materials [5].

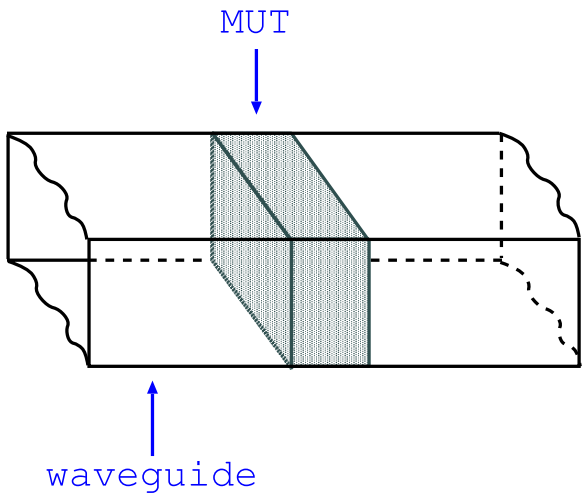

(a) Conventional method.

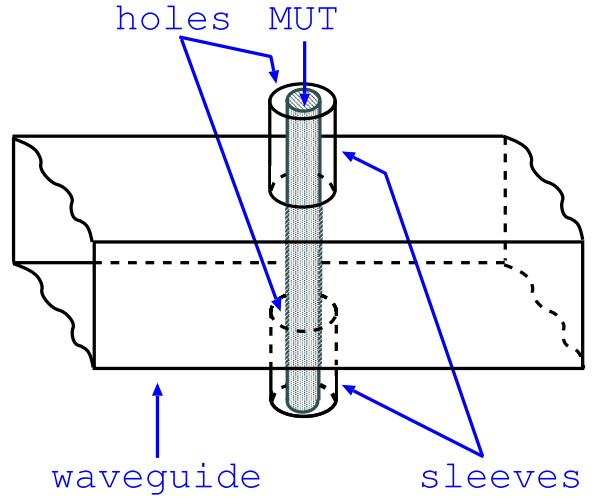

(b) The waveguide-penetration method.

Fig. 1. T/R methods for permittivity measurement.

In this paper, we offer to simplify further the measurement process in the waveguide-penetration method: We propose to eliminate totally the need for system calibration. Instead, we will use only the measurement of two sets of uncalibrated S-parameters, when the MUT is loaded into the waveguide and when it is unloaded from it, to extract correctly the complex permittivity of dielectric materials. Sect. 2 presents the theory behind this new measurement approach, and Sect. 3 presents measurement results that compare favorably with the conventional TRL calibration method. 


\section{Mathematical formulation}

We model the measurement system of the waveguide-penetration method using the 8-term error model and that after VNA's switch error correction [6]. Fig. 2(a) shows the case when the MUT is loaded into the waveguide. Two unknown error networks, $e_{i j}$, are connected to the right and to the left. They represent the unknown hardware components in the system including the test cables, connectors, etc. The MUT in the center is considered reciprocal and symmetric with its S-parameters: $S_{21}=S_{12}=S_{T x}$ and $S_{11}=S_{22}=S_{R x} . S_{T x}$ and $S_{R x}$ are functions of the unknown complex permittivity of the dielectric material, $\varepsilon_{r}=\varepsilon_{r}^{\prime}-j \varepsilon_{r}^{\prime \prime}$, as given by the analysis in [3]. When the MUT is unloaded from the waveguide, i.e. replaced by air material, its S-parameters are replaced by $S_{T a}$ and $S_{R a}$ as shown in Fig. 2(b), while the error terms remain the same. $S_{T a}$ and $S_{R a}$ represent the scattering of the insertion holes, therefore their values are known.

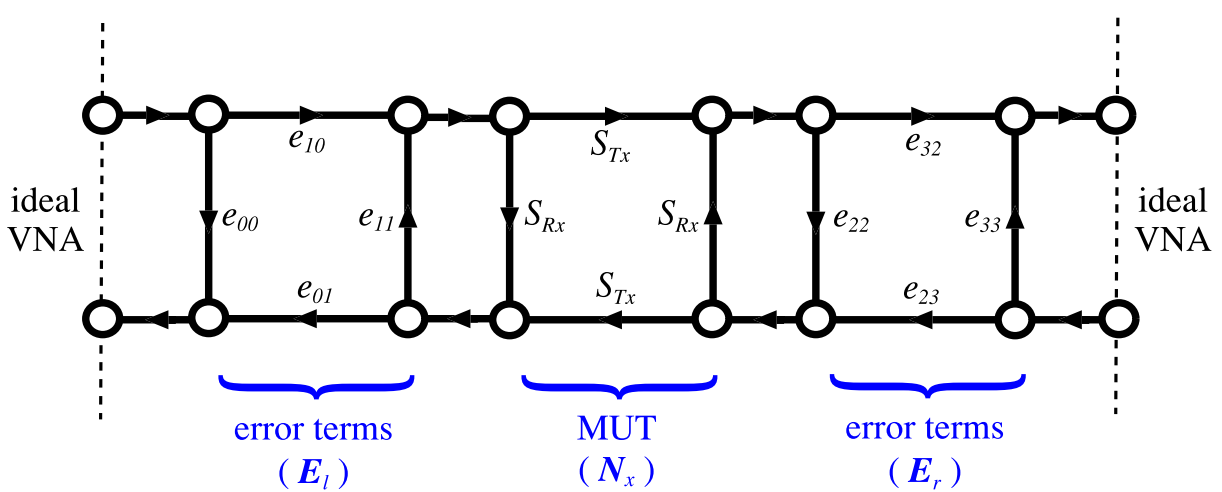

(a) MUT loaded into the waveguide.

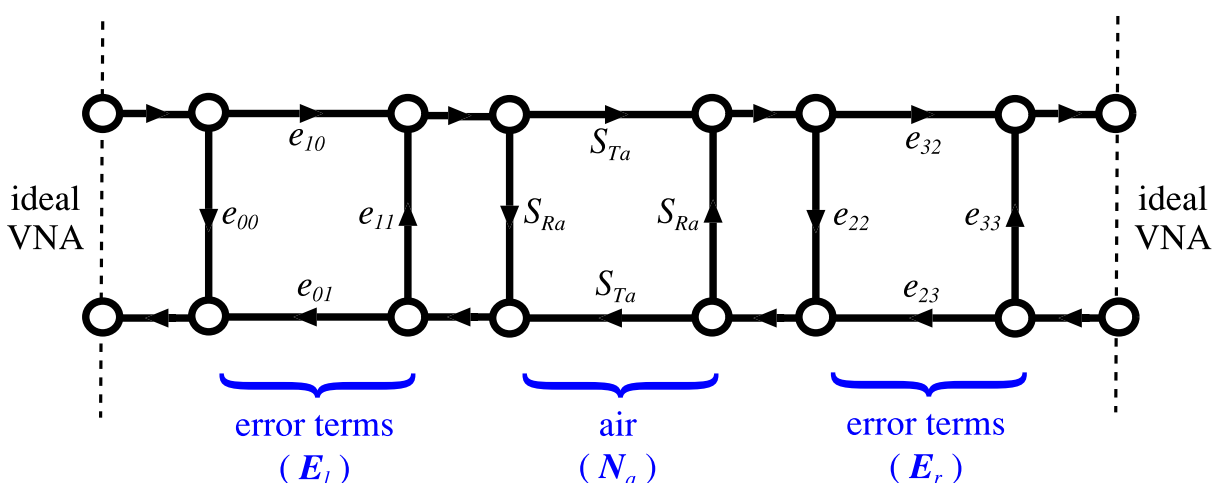

(b) MUT unloaded from the waveguide.

Fig. 2. Flow chart model for the measurement system.

For the mathematical formulation, we will use cascade matrix notation. A cascade matrix is expressed in terms of the S-parameters as

$$
\frac{1}{S_{21}}\left(\begin{array}{cc}
S_{21} S_{12}-S_{11} S_{22} & S_{11} \\
-S_{22} & 1
\end{array}\right) .
$$

Let $\boldsymbol{E}_{l}$ and $\boldsymbol{E}_{r}$ represent the cascade matrices of the unknown left and right error terms in Fig. 2. The MUT is represented by the cascade matrix $\boldsymbol{N}_{x}$ and the air 
material is represented by the cascade matrix $N_{a}$. The measured raw cascade matrix when the MUT is loaded into the waveguide is expressed as

$$
\boldsymbol{M}_{x}=\boldsymbol{E}_{l} \boldsymbol{N}_{x} \boldsymbol{E}_{r},
$$

and when the MUT is unloaded from the waveguide, it is expressed as

$$
\boldsymbol{M}_{a}=\boldsymbol{E}_{l} \boldsymbol{N}_{a} \boldsymbol{E}_{r} .
$$

By multiplying Eq. (2) with the inverse of Eq. (3) on the right, we eliminate the unknown error terms $\boldsymbol{E}_{r}$ as follows:

$$
\boldsymbol{M}_{x} \boldsymbol{M}_{a}^{-1}=\boldsymbol{E}_{l} \boldsymbol{N}_{x} \boldsymbol{N}_{a}^{-1} \boldsymbol{E}_{l}^{-1} .
$$

In Eq. (4), we notice that $\left(\boldsymbol{M}_{x} \boldsymbol{M}_{a}^{-1}\right)$ is a similar transformation of $\left(\boldsymbol{N}_{x} \boldsymbol{N}_{a}^{-1}\right)$ [7]. Using the fact that the trace, which is defined as the sum of the diagonal elements of a matrix, is invariant under the similar transformation, we obtain

$$
\operatorname{Tr}\left(\boldsymbol{M}_{x} \boldsymbol{M}_{a}^{-1}\right)=\operatorname{Tr}\left(\boldsymbol{N}_{x} \boldsymbol{N}_{a}^{-1}\right),
$$

where $\operatorname{Tr}(\cdot)$ represents the trace operator. Therefore, the equal trace operation eliminates the remaining error terms $\boldsymbol{E}_{l}$. Writing $\boldsymbol{N}_{x}$ and $\boldsymbol{N}_{a}^{-1}$ as function of $S_{T x}, S_{R x}$, $S_{T a}$, and $S_{R a}$ in Eq. (5) yields

$$
\operatorname{Tr}\left(\boldsymbol{M}_{x} \boldsymbol{M}_{a}^{-1}\right)=\frac{S_{T x}^{2}-S_{R x}^{2}+S_{T a}^{2}-S_{R a}^{2}+2 S_{R x} S_{R a}}{S_{T x} S_{T a}} .
$$

The left hand side of Eq. (6) is obtained from raw S-parameter measurements. In the right hand side, $S_{T a}$ and $S_{R a}$ are known, while $S_{T x}$ and $S_{R x}$ are function of $\varepsilon_{r}$ of the MUT, the only unknown in the equation. The above equation can therefore be solved for $\varepsilon_{r}$ using iterative root finding algorithms, such as the simplex algorithm for instance.

So from two sets of uncalibrated S-parameter measurements: when the MUT is loaded into the waveguide and when it is unloaded from it, we can determine correctly the complex permittivity of the MUT. The mathematical formulation presented here is a simplification of that presented in [5] in the sense that here we require the measurement of the MUT and the unloaded waveguide only, while [5] required the measurement of two or more reference materials besides the MUT.

\section{Measurement example}

To validate the proposed method, we performed measurement in the X-band $(8.2-12.4 \mathrm{GHz})$ using a WR-90 waveguide $(22.86 \mathrm{~mm} \times 10.16 \mathrm{~mm})$ connected to a VNA (Agilent E8364A, four receivers, 201 points, IF: $100 \mathrm{~Hz}$, no averaging). The holes in the walls of the waveguide have a diameter of $1.81 \mathrm{~mm}$. The MUT is a deionized water at a temperature of $23.5^{\circ} \mathrm{C}$. The de-ionized water is filled inside a glass tube for insertion into the waveguide. The inner diameter of the glass tube is $1.41 \mathrm{~mm}$ and the permittivity of glass is $\varepsilon_{r}=4.31-j 0.03$. We measured the uncalibrated S-parameters both when the MUT is loaded into the waveguide and when it is unloaded from it. The S-parameters are then used in conjunction with Eq. (6) to find iteratively the complex permittivity of water. For comparison, we measured also the complex permittivity of water after TRL calibration. 
Fig. 3 shows the measurement results. Both the real and imaginary parts of the permittivity of water are plotted in red for the proposed method and in blue for the TRL calibration technique. The results of the two methods agree well with each other. The deviation in the measured data, defined as $\left|\varepsilon_{r}^{\text {proposed }}-\varepsilon_{r}^{\mathrm{TRL}}\right|$ at each frequency point, has an RMS value of only 1.2 over the whole frequency band.

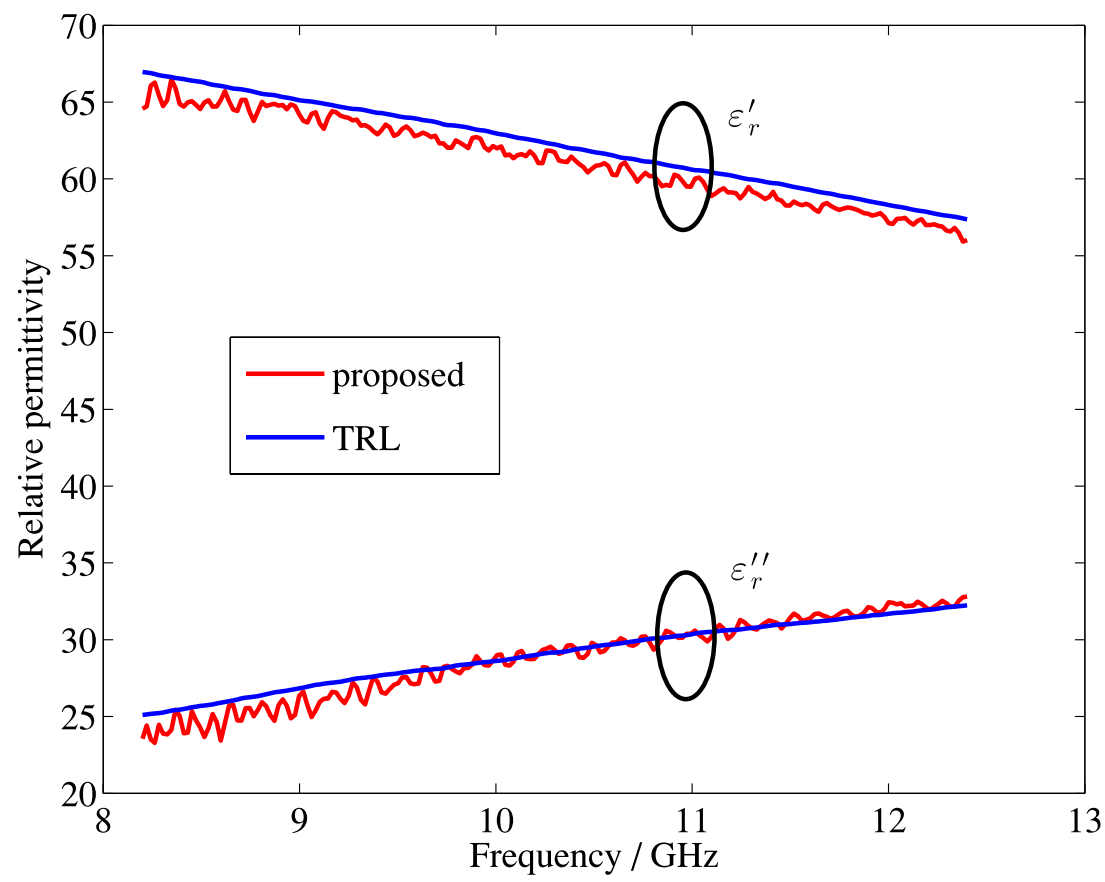

Fig. 3. Permittivity of water in the X-band.

\section{Conclusion}

In this letter, we proposed a method to determine the complex permittivity of dielectric materials in the waveguide-penetration method based on uncalibrated Sparameter measurements. The method only needs to measure two sets of uncalibrated S-parameters: when the MUT is loaded into the waveguide and when the MUT is unloaded from the waveguide. Therefore, it resulted in a simpler measurement process and reduced the time of measurement. The mathematical theory behind the method uses similar matrices and their property of equal traces. First-run experimental results in the X-band demonstrated the validity of this method, and provided results that compares favorably with the conventional TRL calibration method although with slightly lower accuracy. As a next step, an error analysis study is still required to fully understand the performance of the proposed method. 